Divisão de Infecção Hospitalar do Centro de Vigilância Epidemiológica "Prof. Alexandre Vranjac". Coordenadoria de Controle de Doenças da Secretaria de Estado da Saúde de São Paulo - SES-SP

Correspondência | Correspondence: Secretaria de Estado da Saúde de São Paulo Av. Dr. Arnaldo, $3511^{\circ}$ andar sala 135 01246-901 São Paulo, SP, Brasil

E-mail: bepa@saude.sp.gov.br

Texto de difusão técnico-científica da Secretaria de Estado da Saúde de São Paulo.

\section{Sistema de Vigilância Epidemiológica das Infecções Hospitalares do Estado de São Paulo - Análise dos dados de 2005}

\section{Epidemiological Surveillance System for Hospital Infections in the State of São Paulo - Analysis of 2005 data}

Atualmente, as infecções hospitalares (IH) constituem sério problema de saúde pública. Estima-se que a cada dez pacientes hospitalizados, um terá infecção após sua admissão, gerando custos elevados resultantes do aumento do tempo de internação e de intervenções terapêuticas e diagnósticas adicionais.

Estudo de prevalência realizado em hospitais terciários das cinco regiões do Brasil, em 1994, mostrou taxa de IH de 15,5\%. Segundo a Organização Mundial da Saúde (OMS), cabe às autoridades de saúde desenvolver um sistema para monitorar infecções selecionadas e avaliar a efetividade de intervenções.

O Sistema de Vigilância Epidemiológica das Infecções Hospitalares do Estado de São Paulo foi proposto em 2004. Esse sistema valorizou a vigilância de infecções objetivada em unidades críticas e pacientes cirúrgicos, além da seleção de indicadores que permitissem avaliar a qualidade dos processos de atendimento à saúde.

Assim, a partir de 2004, os hospitais do Estado passaram a notificar suas taxas de $\mathrm{IH}$ por meio de planilhas encaminhadas mensalmente por via eletrônica ao Centro de Vigilância Epidemiológica "Prof. Alexandre Vranjac" (CVE) - órgão da Coordenadoria de Controle de Doenças, da Secretaria de Estado da Saúde de São Paulo (CCD/SES-SP). As planilhas são preenchidas de acordo com a complexidade do hospital: planilhas 1,2, 3 e 5 para hospitais gerais e planilha 4 para hospitais especializados (psiquiátrico e de longa permanência). No site do CVE estão disponíveis documentos de orientação para a coleta de dados e preenchimento das planilhas.

Os indicadores epidemiológicos selecionados para hospitais gerais foram: taxa de infecção em cirurgias limpas; densidade de incidência de pneumonia associada à ventilação mecânica $(\mathrm{VM})$; infecção de corrente sanguínea associada a cateter central (CVC) e infecção urinária associada à sonda vesical (SVD); taxas de utilização destes dispositivos invasivos (DI) em Unidade de Terapia Intensiva (UTI) adulto, pediátrica e coronariana; densidade de incidência de pneumonia associada à VM; infecção de corrente sanguínea associada à CVC e taxas de utilização de DI em UTI neonatal, em cada faixa de peso.

Os dados foram consolidados e analisados por meio de planilhas eletrônicas. Os indicadores foram analisados utilizando-se os dados agregados do período (janeiro a dezembro de 2005); ou seja, a soma do número de IH no período, dividida pela soma dos denominadores (número de cirurgias limpas, pacientes-dia, dispositivos invasivos/dia) no período, para cada indicador, multiplicada por mil, no caso das infecções em UTI e em hospitais especializados, ou multiplicados por cem, no caso das infecções de ferida cirúrgica (IFC). As taxas de IH dos hospitais gerais notificantes foram distribuídas em percentis $(10,25,50,75$ e 90$)$.

Com o objetivo de evitar a inclusão de hospitais com denominador extremamente pequeno, foram excluídos das análises hospitais com: menos de 250 cirurgias limpas no período; menos de 500 pacientes-dia em UTI adulto, pediátrica e coronariana; menos de 50 pacientesdia, para cada faixa de peso, em UTI neonatal.

\section{Adesão ao Sistema}

Pelo menos uma planilha de IH no período foi enviada por 534 hospitais, correspondendo a $59,6 \%$ dos cadastrados no Cadastro Nacional dos Estabelecimentos de Saúde (CNES). A média e mediana de hospitais notificantes por mês foram, respectivamente, 398 e 403 (variação de 367-418 hospitais). A Figura 1 mostra o número de hospitais notificantes por mês nos anos de 2004 e 2005. 


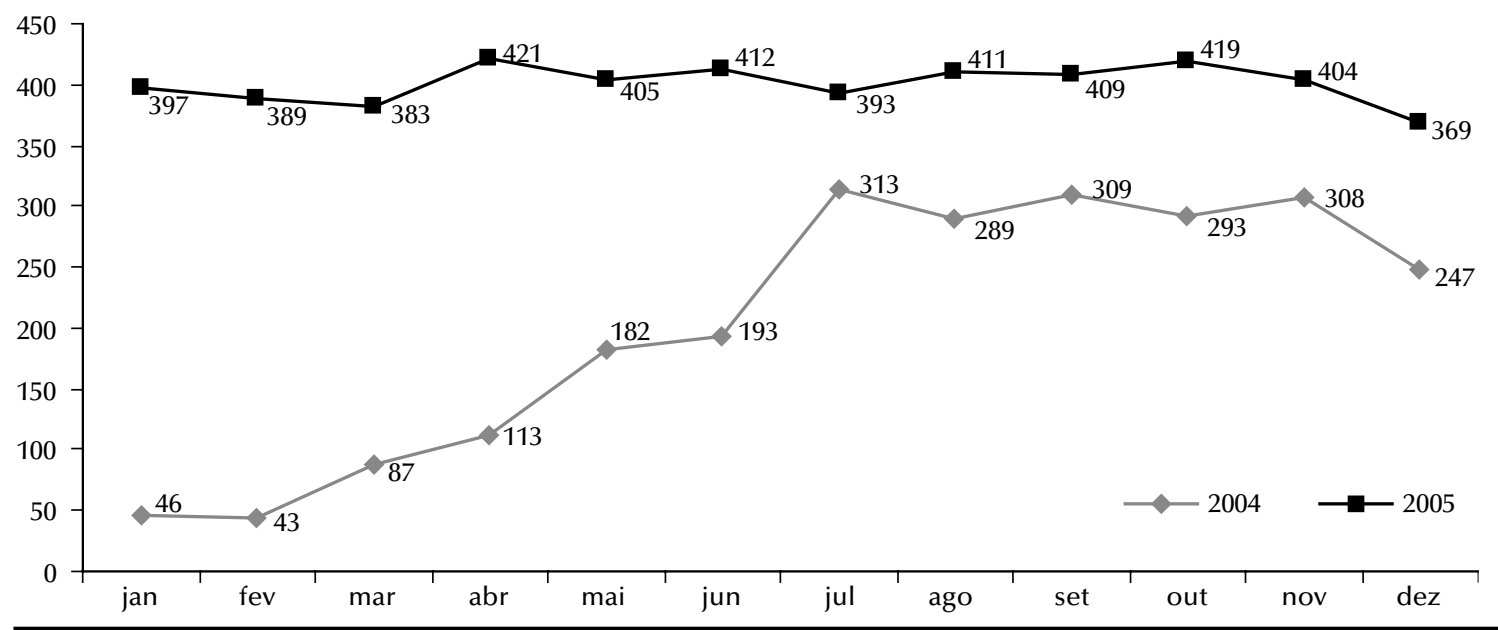

Figura 1. Hospitais notificantes ao Sistema de Vigilância Epidemiológica de Infecções Hospitalares, segundo mês. Secretaria da Saúde, Estado de São Paulo, 2004 e 2005.

\section{Infecções cirúrgicas}

Do total de hospitais notificantes, $85,4 \%$ (456/534) enviaram dados de infecção cirúrgica por meio da planilha 1 e 58,8\% (268/456) informaram que realizam vigilância pós-alta. A distribuição do número de hospitais notificantes, segundo Direção Regional de Saúde, está indicada na Tabela 1.

Foram notificadas 431.446 cirurgias limpas. As Figuras 2 e 3 mostram o número de cirurgias limpas e de hospitais notificantes, segundo especialidade cirúrgica.

$\mathrm{Na}$ análise das taxas de infecção cirúrgica foram incluídos 300 hospitais que notificaram mais de 250 cirurgias limpas em 2005. As Tabelas 2 e 3 apresentam a distribuição das taxas de infecção cirúrgica global e por especialidade cirúrgica, em percentis. Para algumas regionais não foi realizada a distribuição em percentis, pois possuíam menos de dez hospitais com o critério de inclusão adotado para a análise. Entretanto, os dados referentes a essas regionais foram utilizados na análise de percentis do Estado.

\section{Infecções em UTI}

Em todo o Estado, 275 hospitais enviaram dados de infecção em UTI adulto, pediátrica e coronariana (UCO), correspondendo a $51,5 \%$ do total de notificantes em 2005. As Tabelas 4 e 5 mostram o número de hospitais que enviaram a planilha 2 e o número de hospitais por tipo de UTI, segundo a Direção Regional de Saúde.

Foram incluídos na análise das taxas de infecção em UTI adulto, pediátrica e coronariana $213(79,2 \%) 64$ $(71,1 \%)$ e $21(77,8 \%)$ hospitais, respectivamente.

Em UTI adulto, a média de infecções foi de 2.877 pacientes-dia e mediana de 1.961 (variação de 567 a
49.769 pacientes-dia), no período. Em UTI Pediátrica, a média foi de 1.521 pacientes-dia e a mediana de 1.286 (variação: 501 a 7.346 pacientes-dia). Finalmente, a média em UCO foi de 1.502 pacientes-dia e a mediana 1.315 (variação: 571 a 2910 pacientes-dia).

As Tabelas 6,7 e 8 apresentam a distribuição das taxas de infecção em percentis em UTI adulto, Pediátrica e UCO. As Tabelas 9, 10 e 11, as taxas de utilização de dispositivos invasivos (DI) em percentis para essas unidades.

\section{Infecções em UTI neonatal}

O número de hospitais que enviou planilha 3 foi de 124 , que corresponde a $23,2 \%$ do total de notificantes ao Sistema de Vigilância das IH do Estado de São Paulo (Tabela 12).

Foram incluídos 110 hospitais para cálculo das taxas de IH por faixa de peso. Um mesmo hospital pode ter sido incluído na análise de taxas de mais de uma faixa de peso do recém-nascido. A distribuição do número de notificantes da planilha 3 incluídos na análise, por faixa de peso, foi de 83 hospitais para a faixa de peso $<1.000 \mathrm{~g}, 105$ para a faixa de $1.001 \mathrm{a} 1500 \mathrm{~g}, 110$ para $1.501 \mathrm{~g}$ a $2.500 \mathrm{~g}$ e $109 \mathrm{para}>2.500 \mathrm{~g}$.

Nas Tabelas 13 e 14 são apresentadas as densidades de incidência de infecção associada a dispositivos invasivos, distribuídas em percentis, por faixa de peso em UTI neonatal. As tabelas 15 e 16 apresentam a distribuição das taxas de utilização de DI em percentis por faixa de peso.

\section{Hemocultura}

Na análise da distribuição dos microrganismos isolados em hemoculturas em UTI não foi utilizado qualquer 


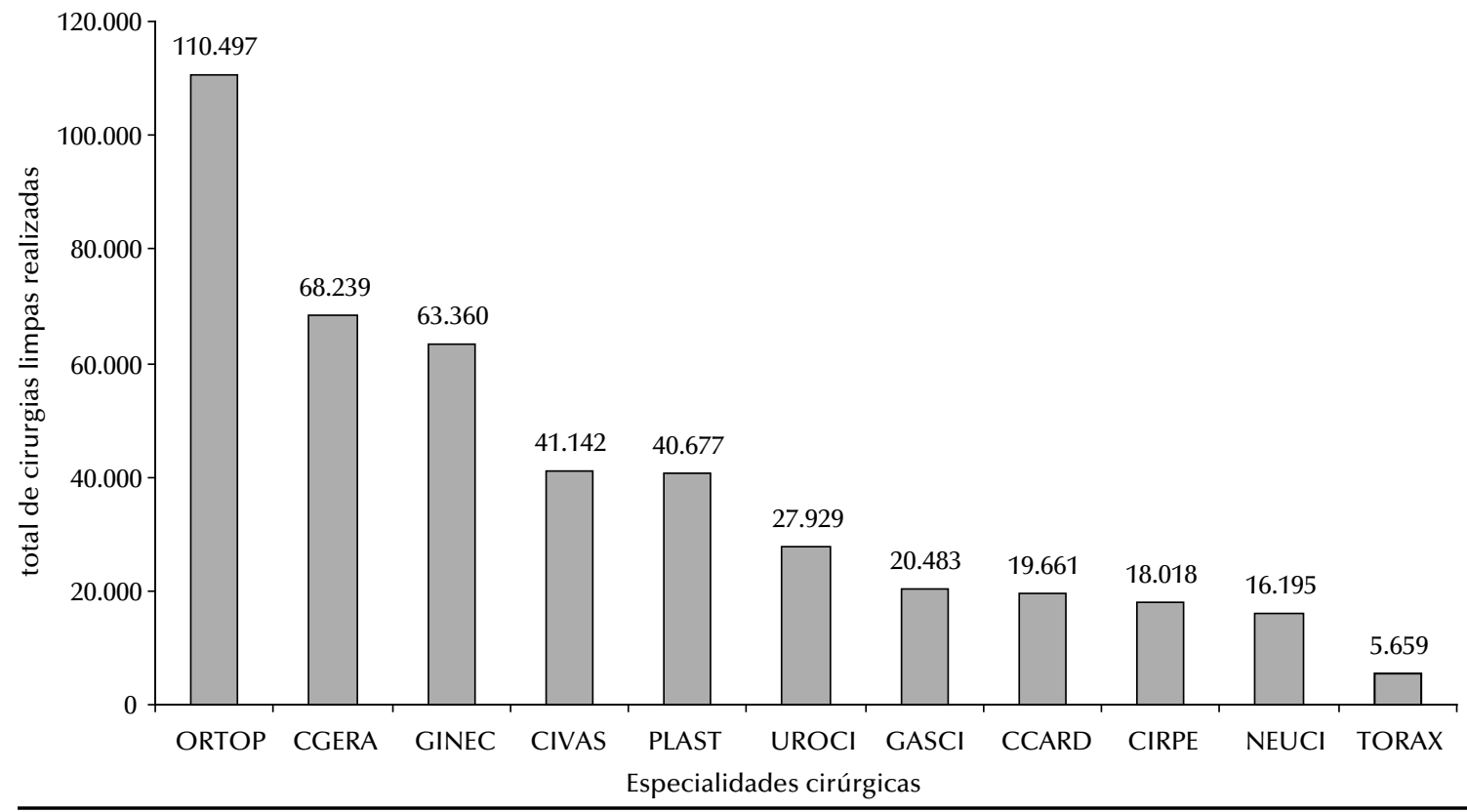

Figura 2. Número de cirurgias limpas notificadas ao Sistema de Vigilância Epidemiológica das Infecções Hospitalares, por especialidade cirúrgica. Secretaria da Saúde, Estado de São Paulo, 2005.

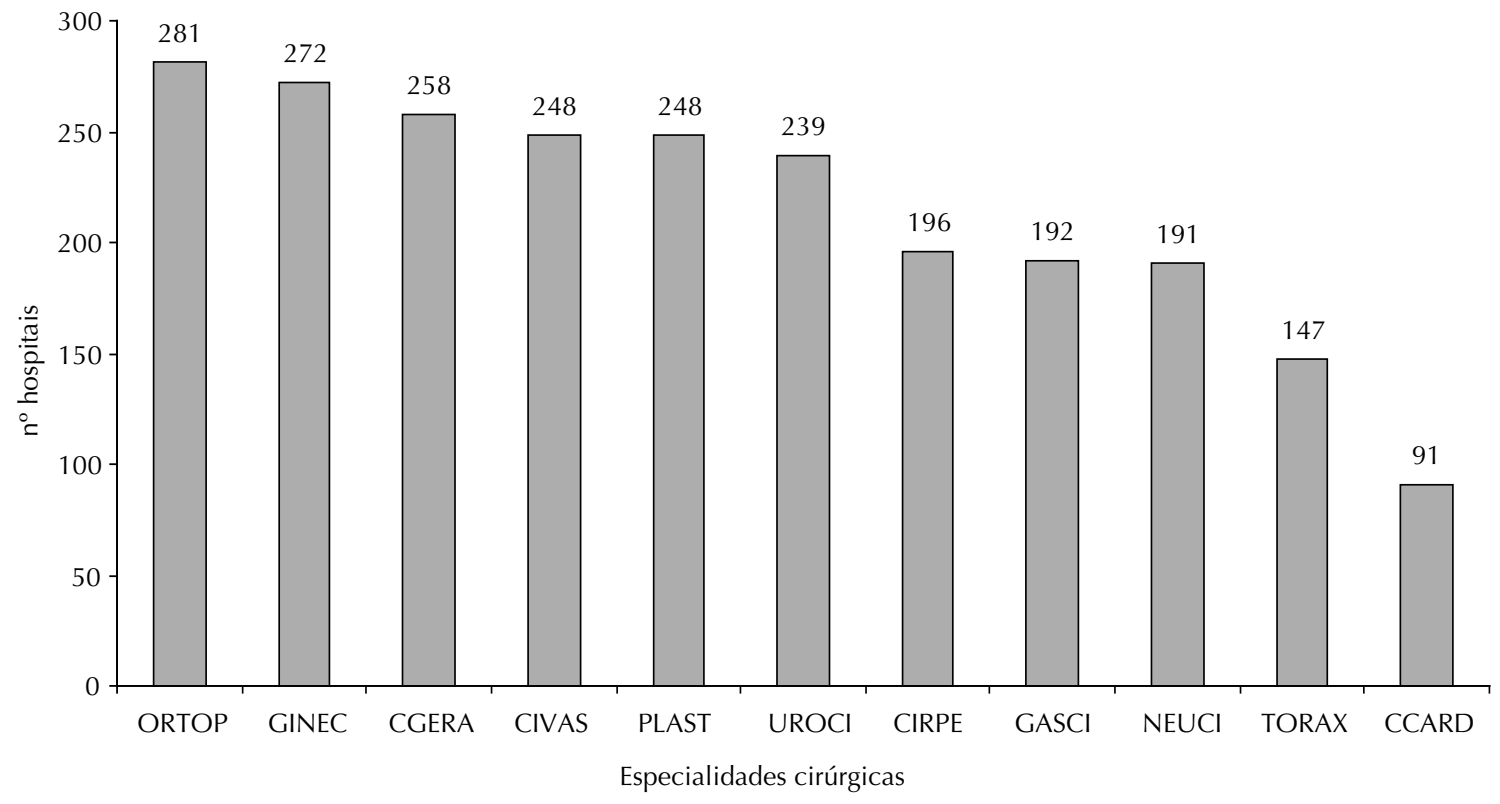

Figura 3. Número de hospitais notificantes de infecções hospitalares ao Sistema de Vigilância Epidemiológica, por especialidade cirúrgica Secretaria da Saúde. Estado de São Paulo, 2005.

critério de exclusão, por tratar-se de uma avaliação qualitativa de dados. Desse modo, os dados de todos os hospitais notificantes foram analisados.

Foram notificados 8.492 pacientes com IH e hemocultura positiva. A Tabela 17 apresenta a distribuição percentual dos microrganismos isolados em hemoculturas e a Tabela 18, o seu perfil de resistência.

\section{COMENTÁRIOS}

Quando comparado a 2004, em 2005 houve aumento do número de hospitais notificantes ao Sistema de Vigilância das Infecções Hospitalares do Estado de São Paulo. Da mesma forma, foi maior o número de hospitais notificantes por mês com regularidade de envio de dados. 
Conforme verificado em 2004, a maioria dos hospitais do Estado realiza procedimentos cirúrgicos $(85,4 \%)$. A mediana das taxas de infecção cirúrgica apresentou-se abaixo do esperado, quando considerada a alta taxa de vigilância pós-alta referida pelos hospitais que realizam procedimentos cirúrgicos. O sistema tem como objetivo diminuir o risco de subnotificação, uma vez que $12 \%$ a $84 \%$ das infecções cirúrgicas ocorrem após a alta do paciente. A taxa de infecção cirúrgica do Estado sugere que, além da subnotificação, este tipo de vigilância não tem sido realizado.

A taxa de infecção mais elevada em cirurgia cardíaca pode ser explicada pelo fato de os pacientes, geralmente, retornarem ao serviço de origem para tratamento de infecção após o procedimento. Com isso, é mais fácil a recuperação das taxas de infecção.

Os dados solicitados pela planilha 2 foram estratificados em UTI adulto, pediátrica e coronariana. A estratificação tinha por objetivo facilitar a notificação e possibilitar a comparação de dados de acordo com o perfil de atendimento das unidades.

Para UTI neonatal a taxa de utilização de DI é mais alta quanto menor a faixa de peso ao nascer, indicando maior gravidade dos bebês com menor peso.
Staphylococcus epidermidis e outros Staphylococcus coagulase negativa foram os microrganismos mais freqüentemente isolados em pacientes com $\mathrm{IH}$ e hemocultura positiva. Este dado deve ser avaliado com cuidado, pois há dúvidas se esses agentes podem ser realmente considerados como etiológicos das infecções ou se estão ocorrendo falhas nos procedimentos de coleta de hemoculturas.

A análise do perfil de resistência dos microrganismos isolados em hemocultura mostra que este é um problema emergente e merece atenção com ações governamentais específicas.

O aumento da adesão ao Sistema de Vigilância Epidemiológica das IH do Estado de São Paulo mostra a efetividade do trabalho contínuo de sensibilização dos hospitais, desenvolvido pela Divisão de Infecção Hospitalar em parceria com as Regionais de Saúde e os municípios.

O estímulo à notificação deve ser mantido para que a notificação seja crescente, aumentando a consistência dos dados. Além disso, serão realizados treinamentos com enfoque nos critérios diagnósticos e preenchimento das planilhas para melhorar a qualidade dos dados, permitindo a comparação de dados mais homogêneos. 
Tabela 1. Hospitais notificantes ao Sistema de Vigilância das Infecções Hospitalares, segundo regional de saúde, planilha 1 e realização de vigilância pós-alta. Secretaria da Saúde do Estado de São Paulo, 2005.

\begin{tabular}{|c|c|c|c|c|c|}
\hline \multirow[t]{2}{*}{ Regional de saúde } & \multirow{2}{*}{$\begin{array}{c}\text { Hospitais notificantes } \\
2005 \\
\text { N }\end{array}$} & \multicolumn{2}{|c|}{$\begin{array}{l}\text { Hospitais que enviaram } \\
\text { planilha } 1\end{array}$} & \multicolumn{2}{|c|}{$\begin{array}{l}\text { Hospitais que realizam } \\
\text { vigilância pós-alta }\end{array}$} \\
\hline & & $\mathrm{N}$ & $\%$ & $\mathrm{~N}$ & $\%$ \\
\hline I - São Paulo & 52 & 39 & 75,0 & 19 & 48,7 \\
\hline II - Santo André & 32 & 27 & 84,4 & 15 & 55,6 \\
\hline III - Mogi das Cruzes & 24 & 22 & 91,7 & 6 & 27,3 \\
\hline IV - Franco da Rocha & 2 & 2 & 100,0 & 1 & 50,0 \\
\hline V-Osasco & 3 & 2 & 66,7 & 1 & 50,0 \\
\hline VI - Araçatuba & 32 & 28 & 87,5 & 18 & 64,3 \\
\hline VII - Araraquara & 20 & 18 & 90,0 & 12 & 66,7 \\
\hline VIII - Assis & 13 & 12 & 92,3 & 7 & 58,3 \\
\hline IX - Barretos & 16 & 15 & 93,8 & 10 & 66,7 \\
\hline$X$ - Bauru & 35 & 31 & 88,6 & 21 & 67,7 \\
\hline XI - Botucatu & 22 & 19 & 86,4 & 14 & 73,7 \\
\hline XII - Campinas & 41 & 37 & 90,2 & 14 & 37,8 \\
\hline XIII - Franca & 1 & 1 & 100,0 & 0 & 0,0 \\
\hline XIV - Marília & 22 & 15 & 68,2 & 11 & 73,3 \\
\hline XV - Piracicaba & 25 & 20 & 80,0 & 15 & 75,0 \\
\hline XVI - Presidente Prudente & 28 & 24 & 85,7 & 9 & 37,5 \\
\hline XVII - Registro & 1 & 1 & 100,0 & 0 & 0,0 \\
\hline XVIII - Ribeirão Preto & 25 & 24 & 96,0 & 21 & 87,5 \\
\hline XIX - Santos & 18 & 17 & 94,4 & 7 & 41,2 \\
\hline XX - São João da Boa Vista & 20 & 15 & 75,0 & 12 & 80,0 \\
\hline XXI - São José dos Campos & 27 & 24 & 88,9 & 16 & 66,7 \\
\hline XXII - São José do Rio Preto & 36 & 34 & 94,4 & 26 & 76,5 \\
\hline XXIII - Sorocaba & 29 & 22 & 75,9 & 10 & 45,5 \\
\hline XXIV - Taubaté & 10 & 7 & 70,0 & 3 & 42,9 \\
\hline Total & 534 & 456 & 85,4 & 268 & 58,8 \\
\hline
\end{tabular}


Tabela 2. Taxas de infecção cirúrgica em percentis dos hospitais que notificaram ao Sistema de Vigilância Epidemiológica das Infecções Hospitalares, segundo as regional de saúde. Secretaria da Saúde, . Estado de São Paulo, 2005.

\begin{tabular}{|c|c|c|c|c|c|c|c|}
\hline \multirow{2}{*}{ Regional de saúde } & \multirow{2}{*}{$\begin{array}{c}\text { Hospitais* } \\
\mathrm{N}\end{array}$} & \multicolumn{5}{|c|}{ Percentil } & \multirow{2}{*}{$\begin{array}{l}\text { Valor } \\
\text { máximo }\end{array}$} \\
\hline & & 10 & 25 & 50 & 75 & 90 & \\
\hline I & 32 & 0,24 & 0,52 & 0,94 & 1,74 & 5,01 & 8,49 \\
\hline II & 22 & 0,16 & 0,36 & 0,52 & 0,78 & 2,63 & 4,72 \\
\hline III & 14 & 0,00 & 0,13 & 0,36 & 0,93 & 1,52 & 5,64 \\
\hline IV & 1 & & & & & & \\
\hline V & 1 & & & & & & \\
\hline VI & 13 & 0,00 & 0,00 & 0,00 & 0,22 & 0,31 & 0,79 \\
\hline VII & 12 & 0,01 & 0,52 & 1,54 & 2,43 & 2,81 & 3,01 \\
\hline VIII & 8 & & & & & & \\
\hline IX & 6 & & & & & & \\
\hline$x$ & 17 & 0,00 & 0,00 & 0,38 & 0,81 & 1,10 & 1,84 \\
\hline XI & 8 & & & & & & \\
\hline XII & 25 & 0,00 & 0,05 & 0,53 & 1,03 & 2,20 & 4,70 \\
\hline XIII & 1 & & & & & & \\
\hline XIV & 9 & & & & & & \\
\hline$x V$ & 19 & 0,00 & 0,19 & 0,42 & 1,37 & 1,98 & 3,04 \\
\hline XVI & 14 & 0,00 & 0,00 & 0,27 & 1,05 & 1,66 & 4,01 \\
\hline XVII & 0 & & & & & & \\
\hline XVIII & 12 & 0,01 & 0,55 & 1,37 & 1,62 & 2,21 & 2,58 \\
\hline XIX & 15 & 0,00 & 0,05 & 0,41 & 1,23 & 1,76 & 3,83 \\
\hline$X X$ & 10 & 0,00 & 0,14 & 0,26 & 1,87 & 3,34 & 4,76 \\
\hline XXI & 19 & 0,00 & 0,00 & 0,66 & 1,10 & 1,54 & 2,70 \\
\hline XXII & 17 & 0,00 & 0,00 & 0,11 & 0,84 & 1,16 & 2,38 \\
\hline XXIII & 19 & 0,07 & 0,27 & 0,68 & 1,17 & 1,63 & 9,36 \\
\hline XXIV & 6 & & & & & & \\
\hline Total & 300 & 0,00 & 0,07 & 0,57 & 1,25 & 2,59 & 9,36 \\
\hline
\end{tabular}

*Com mais de 250 cirurgias realizadas

Tabela 3. Taxas de infecção por especialidade cirúrgica em percentis dos hospitais que notificaram ao Sistema de Vigilância Epidemiológica das Infecções Hospitalares. Secretaria da Saúde, Estado de São Paulo, 2005.

\begin{tabular}{lcccccc}
\hline Especialidade & Hospitais* & \multicolumn{5}{c}{ Taxa de infecção Percentil } \\
cirúrgica & $\mathrm{N}$ & 10 & 25 & 50 & 75 & 90 \\
\hline Cardíaca & 91 & 0,00 & 0,00 & 1,91 & 6,56 & 10,14 \\
Geral & 258 & 0,00 & 0,00 & 0,00 & 1,27 & 3,16 \\
Pediátrica & 196 & 0,00 & 0,00 & 0,00 & 0,00 & 1,01 \\
Vascular & 248 & 0,00 & 0,00 & 0,00 & 0,61 & 2,90 \\
Gastrocirurgia & 192 & 0,00 & 0,00 & 0,00 & 0,73 & 2,93 \\
Ginecologia & 272 & 0,00 & 0,00 & 0,00 & 0,65 & 1,88 \\
Neurocirurgia & 190 & 0,00 & 0,00 & 0,00 & 3,23 & 5,95 \\
Ortopedia & 281 & 0,00 & 0,00 & 0,00 & 1,10 & 2,61 \\
Plástica & 248 & 0,00 & 0,00 & 0,00 & 0,00 & 0,98 \\
Torácia & 147 & 0,00 & 0,00 & 0,00 & 0,00 & 2,39 \\
Urológica & 239 & 0,00 & 0,00 & 0,00 & 0,00 & 2,11 \\
\hline
\end{tabular}

*Com mais de 250 cirurgias realizadas 
Tabela 4. Hospitais que notificaram ao Sistema de Vigilância Epidemiológica das Infecções Hospitalares, segundo planilha 2 e regional de saúde. Secretaria da Saúde, Estado de São Paulo, 2005.

\begin{tabular}{|c|c|c|c|}
\hline \multirow{2}{*}{$\begin{array}{l}\text { Regional } \\
\text { de saúde }\end{array}$} & \multirow{2}{*}{$\begin{array}{c}\text { Hospitais } \\
\text { notificantes } 2005 \\
\text { N }\end{array}$} & \multicolumn{2}{|c|}{$\begin{array}{l}\text { Hospitais que } \\
\text { enviaram planilha } 2 *\end{array}$} \\
\hline & & $\mathrm{N}$ & $\%$ \\
\hline I & 52 & 47 & 90,4 \\
\hline II & 32 & 25 & 78,1 \\
\hline III & 24 & 20 & 83,3 \\
\hline IV & 2 & 1 & 50,0 \\
\hline V & 3 & 3 & 100,0 \\
\hline VI & 32 & 8 & 25,0 \\
\hline VII & 20 & 8 & 40,0 \\
\hline VIII & 13 & 6 & 46,2 \\
\hline IX & 16 & 6 & 37,5 \\
\hline$x$ & 35 & 15 & 42,9 \\
\hline XI & 22 & 3 & 13,6 \\
\hline XII & 41 & 31 & 75,6 \\
\hline XIII & 1 & 1 & 100,0 \\
\hline XIV & 22 & 6 & 27,3 \\
\hline$X V$ & 25 & 11 & 44,0 \\
\hline XVI & 28 & 7 & 25,0 \\
\hline XVII & 1 & 0 & 0,0 \\
\hline XVIII & 25 & 13 & 52,0 \\
\hline XIX & 18 & 12 & 66,7 \\
\hline$X X$ & 20 & 6 & 30,0 \\
\hline XXI & 27 & 13 & 48,1 \\
\hline XXII & 36 & 11 & 30,6 \\
\hline XXIII & 29 & 16 & 55,2 \\
\hline XXIV & 10 & 6 & 60,0 \\
\hline Total & 534 & 275 & 51,5 \\
\hline
\end{tabular}

*UTI adulto, UTI pediátrica, UTI coronariana
Tabela 5. Hospitais que notificaram ao Sistema de Vigilância Epidemiológica das Infecções Hospitalares, segundo tipo de UTI e regional de saúde. Secretaria da Saúde, Estado de São Paulo, 2005

\begin{tabular}{|c|c|c|c|}
\hline \multirow{2}{*}{$\begin{array}{l}\text { Regional } \\
\text { de saúde }\end{array}$} & \multicolumn{3}{|c|}{ Tipo de UTI } \\
\hline & Adulto & Coronariana & Pediátrica \\
\hline 1 & 43 & 10 & 28 \\
\hline II & 25 & 0 & 6 \\
\hline III & 20 & 1 & 8 \\
\hline IV & 1 & 0 & 1 \\
\hline V & 3 & 0 & 2 \\
\hline $\mathrm{VI}$ & 8 & 1 & 1 \\
\hline VII & 8 & 1 & 4 \\
\hline VIII & 6 & 0 & 1 \\
\hline IX & 6 & 0 & 1 \\
\hline$x$ & 15 & 2 & 4 \\
\hline$X I$ & 3 & 0 & 1 \\
\hline XII & 31 & 3 & 6 \\
\hline XIII & 1 & 0 & 1 \\
\hline XIV & 6 & 0 & 1 \\
\hline$X V$ & 11 & 2 & 2 \\
\hline $\mathrm{XVI}$ & 7 & 1 & 1 \\
\hline XVII & 0 & 0 & 0 \\
\hline XVIII & 13 & 1 & 4 \\
\hline XIX & 12 & 2 & 5 \\
\hline$X X$ & 6 & 0 & 0 \\
\hline$X X I$ & 12 & 1 & 4 \\
\hline XXII & 11 & 2 & 2 \\
\hline XXIII & 15 & 0 & 5 \\
\hline XXIV & 6 & 0 & 2 \\
\hline Total & 269 & 27 & 90 \\
\hline
\end{tabular}

Tabela 6. Distribuição das taxas de infecção associadas a dispositivos invasivos em UTI adulto, em percentis. Secretaria da Saúde, Estado de São Paulo, 2005.

\begin{tabular}{lcccccc}
\hline & \multicolumn{5}{c}{ Densidade de incidência (por 1.000 DI-dia) } \\
Infecção sob vigilância & 10 & 25 & 50 & 75 & 90 & Variação \\
\hline $\begin{array}{l}\text { Pneumonia associada à } \\
\text { ventilação mecânica }\end{array}$ & 0,37 & 10,79 & 19,40 & 27,70 & 43,60 & $0,0-48,67$ \\
$\begin{array}{l}\text { Infecção de corrente sanguínea } \\
\text { associada à cateter central }\end{array}$ & 0,00 & 1,08 & 4,97 & 4,97 & 9,19 & $0,0-28,28$ \\
$\begin{array}{l}\text { Infecção de trato urinário } \\
\text { associada à sonda vesical }\end{array}$ & 0,00 & 2,96 & 7,27 & 7,27 & 18,75 & $0,0-32,18$ \\
\hline
\end{tabular}

DI: Dispositivo invasivo 
Tabela 7. Distribuição das taxas de infecção associadas a dispositivos invasivos em UTI pediátrica, em percentis. Secretaria da Saúde, Estado de São Paulo, 2005.

\begin{tabular}{|c|c|c|c|c|c|c|}
\hline \multirow{3}{*}{ Infecção sob vigilância } & \multicolumn{6}{|c|}{ Densidade de incidência (por 1.000 DI-dia) } \\
\hline & \multicolumn{5}{|c|}{ Percentil } & \multirow{2}{*}{ Variação } \\
\hline & 10 & 25 & 50 & 75 & 90 & \\
\hline $\begin{array}{l}\text { Pneumonia associada à } \\
\text { ventilação mecânica }\end{array}$ & 0,00 & 3,26 & 7,43 & 13,53 & 19,35 & $0,0-46,63$ \\
\hline $\begin{array}{l}\text { Infecção de corrente sanguínea } \\
\text { associada à cateter central }\end{array}$ & 0,00 & 2,34 & 9,58 & 16,42 & 22,62 & $0,0-39,06$ \\
\hline $\begin{array}{l}\text { Infecção de trato urinário } \\
\text { associada à sonda vesical }\end{array}$ & 0,00 & 0,00 & 2,58 & 8,64 & 21,85 & $0,0-28,57$ \\
\hline
\end{tabular}

Tabela 8. Distribuição das taxas de infecção associadas a dispositivos invasivos em UTI coronariana, em percentis. Secretaria da Saúde, Estado de São Paulo, 2005.

\begin{tabular}{|c|c|c|c|c|c|c|}
\hline \multirow{3}{*}{ Infecção sob vigilância } & \multicolumn{6}{|c|}{ Densidade de incidência (por 1.000 DI-dia) } \\
\hline & \multicolumn{5}{|c|}{ Percentil } & \multirow{2}{*}{ Variação } \\
\hline & 10 & 25 & 50 & 75 & 90 & \\
\hline $\begin{array}{l}\text { Pneumonia associada à } \\
\text { ventilação mecânica }\end{array}$ & 5,38 & 12,20 & 20,65 & 28,17 & 47,17 & $0,0-80,15$ \\
\hline $\begin{array}{l}\text { Infecção de corrente sanguínea } \\
\text { associada à cateter central }\end{array}$ & 0,00 & 0,00 & 0,93 & 2,23 & 8,11 & $0,0-11,13$ \\
\hline $\begin{array}{l}\text { Infecção de trato urinário } \\
\text { associada à sonda vesical }\end{array}$ & 1,05 & 3,27 & 4,66 & 10,20 & 12,58 & $0,0-12,21$ \\
\hline
\end{tabular}

Tabela 9. Distribuição das taxas de utilização de dispositivo invasivos em percentis em UTI adulto. Secretaria da Saúde, Estado de São Paulo, 2005.

\begin{tabular}{lccccc}
\hline \multirow{2}{*}{ Dispositivo invasivo } & \multicolumn{5}{c}{ Taxa de utilização } \\
& 10 & 25 & 50 & 75 & 90 \\
\hline Ventilação mecânica & 19,60 & 30,83 & 42,04 & 54,18 & 64,31 \\
Cateter central & 20,67 & 31,89 & 48,21 & 64,54 & 75,89 \\
Sonda vesical & 41,01 & 56,45 & 67,70 & 79,29 & 86,16 \\
\hline
\end{tabular}

Tabela 10. Distribuição das taxas de utilização de dispositivos invasivos em percentis em UTI pediátrica. Secretaria da Saúde, Estado de São Paulo, 2005.

\begin{tabular}{lccccc}
\hline & \multicolumn{5}{c}{ Taxa de utilização } \\
Dispositivo invasivo & 10 & 25 & 50 & 75 & 90 \\
\hline Ventilação mecânica & 18,24 & 28,74 & 40,93 & 53,20 & 64,67 \\
Cateter central & 10,72 & 23,57 & 36,41 & 46,22 & 65,12 \\
Sonda vesical & 2,19 & 7,22 & 12,05 & 19,66 & 30,92 \\
\hline
\end{tabular}

Tabela 11. Distribuição das taxas de utilização de dispositivos invasivos em percentis em UTI coronariana. Secretaria da Saúde, Estado de São Paulo, 2005.

\begin{tabular}{lccccc}
\hline & \multicolumn{5}{c}{ Taxa de utilização } \\
Dispositivo invasivo & \multicolumn{5}{c}{ Percentil } \\
& 10 & 25 & 50 & 75 & 90 \\
\hline Ventilação mecânica & 9,66 & 12,89 & 18,56 & 26,22 & 30,43 \\
Cateter central & 18,33 & 27,95 & 34,28 & 44,66 & 50,71 \\
Sonda vesical & 24,18 & 33,97 & 42,41 & 55,40 & 61,44 \\
\hline
\end{tabular}


Tabela 12. Hospitais que notirficaram ao Sistema deVigilância Epidemiológica das Infecções Hospitalares, segundo planilha 3 e regional de saúde. Secretaria da Saúde, Estado de São Paulo, 2005.

\begin{tabular}{|c|c|c|c|}
\hline \multirow{2}{*}{$\begin{array}{l}\text { Regional } \\
\text { de saúde }\end{array}$} & \multirow{2}{*}{$\begin{array}{c}\text { Hospitais } \\
\text { notificantes } 2005 \\
\text { N }\end{array}$} & \multicolumn{2}{|c|}{$\begin{array}{c}\text { Hospitais que } \\
\text { enviaram planilha } 3\end{array}$} \\
\hline & & $\mathrm{N}$ & $\%$ \\
\hline I & 52 & 19 & 36,5 \\
\hline II & 32 & 12 & 37,5 \\
\hline III & 24 & 13 & 54,2 \\
\hline IV & 2 & 1 & 50,0 \\
\hline V & 3 & 3 & 100,0 \\
\hline $\mathrm{VI}$ & 32 & 1 & 3,1 \\
\hline VII & 20 & 4 & 20,0 \\
\hline VIII & 13 & 2 & 15,4 \\
\hline IX & 16 & 1 & 6,3 \\
\hline$x$ & 35 & 4 & 11,4 \\
\hline XI & 22 & 1 & 4,5 \\
\hline XII & 41 & 15 & 36,6 \\
\hline XIII & 1 & 1 & 100,0 \\
\hline XIV & 22 & 2 & 9,1 \\
\hline$X V$ & 25 & 5 & 20,0 \\
\hline XVI & 28 & 5 & 17,9 \\
\hline XVII & 1 & 0 & 0,0 \\
\hline XVIII & 25 & 7 & 28,0 \\
\hline XIX & 18 & 9 & 50,0 \\
\hline$X X$ & 20 & 1 & 5,0 \\
\hline XXI & 27 & 5 & 18,5 \\
\hline XXII & 36 & 5 & 13,9 \\
\hline XXIII & 29 & 4 & 13,8 \\
\hline XXIV & 10 & 4 & 40,0 \\
\hline Total & 534 & 124 & 23,2 \\
\hline
\end{tabular}

Tabela 13. Taxas de pneumonia associada à ventilação mecânica em UTI neonatal, segundo faixa de peso. Secretaria da Saúde, Estado de São Paulo, 2005.

\begin{tabular}{lcccccc}
\hline \multicolumn{5}{c}{$\begin{array}{c}\text { Densidade de incidência de pneumonia } \\
\text { (x1.000 VM-dia) } \\
\text { Percentil }\end{array}$} \\
& 10 & 25 & 50 & 75 & 90 \\
\hline Faixa de peso & & & & & \\
$\leq 1.000 \mathrm{~g}$ & 0,00 & 0,00 & 7,76 & 17,73 & 28,47 \\
$1.001-1.500 \mathrm{~g}$ & 0,00 & 0,00 & 5,73 & 23,06 & 45,13 \\
$1.501-2.500 \mathrm{~g}$ & 0,00 & 0,00 & 0,00 & 14,60 & 59,57 \\
$>2.500 \mathrm{~g}$ & 0,00 & 0,00 & 0,00 & 15,00 & 36,24 \\
\hline
\end{tabular}

VM: ventilação mecânica
Tabela 14. Taxas de infecção de corrente sanguínea associada a cateter central em UTI neonatal, segundo faixa de peso. Secretaria da Saúde, Estado de São Paulo, 2005.

\begin{tabular}{lccccc}
\hline & \multicolumn{5}{c}{ Densidade de incidência de infecção } \\
& 10 & 25 & 50 & 75 & 90 \\
\hline Faixas de peso & & & & & \\
$\leq 1.000 \mathrm{~g}$ & 0,00 & 0,00 & 14,29 & 31,91 & 49,50 \\
$1.001-1.500 \mathrm{~g}$ & 0,00 & 0,00 & 13,89 & 35,29 & 64,49 \\
$1.501-2.500 \mathrm{~g}$ & 0,00 & 0,00 & 13,16 & 36,30 & 60,29 \\
$>2.500 \mathrm{~g}$ & 0,00 & 0,00 & 8,67 & 29,63 & 55,56 \\
\hline
\end{tabular}

Tabela 15. Taxas de utilização de ventilação mecânica em UTI neonatal, segundo faixa de peso. Secretaria da Saúde, Estado de São Paulo, 2005.

\begin{tabular}{|c|c|c|c|c|c|}
\hline & \multicolumn{5}{|c|}{$\begin{array}{c}\text { Taxa de utilização de ventilação me- } \\
\text { cânica } \\
\text { Percentil }\end{array}$} \\
\hline & 10 & 25 & 50 & 75 & 90 \\
\hline \multicolumn{6}{|l|}{ Faixa de peso } \\
\hline$\leq 1.000 \mathrm{~g}$ & 30,21 & 44,14 & 61,05 & 77,06 & 84,75 \\
\hline $1.001-1.500 \mathrm{~g}$ & 11,50 & 16,59 & 29,30 & 44,90 & 66,08 \\
\hline $1.501-2.500 \mathrm{~g}$ & 4,78 & 9,66 & 17,90 & 33,12 & 45,29 \\
\hline$>2.500 \mathrm{~g}$ & 5,17 & 9,60 & 21,43 & 37,38 & 56,48 \\
\hline
\end{tabular}

Tabela 16. Taxas de utilização de cateter central em UTI neonatal, segundo faixa de peso. Secretaria da Saúde, Estado de São Paulo, 2005.

\begin{tabular}{lrrrrr}
\hline & \multicolumn{5}{c}{ Taxa de utilização de cateter central } \\
& \multicolumn{5}{c}{ Percentil } \\
& 10 & \multicolumn{1}{c}{25} & 50 & 75 & 90 \\
\hline Faixa de peso & & & & & \\
$\leq 1.000 \mathrm{~g}$ & 25,01 & 38,17 & 57,67 & 76,24 & 86,51 \\
$1.001-1.500 \mathrm{~g}$ & 9,93 & 20,40 & 39,32 & 62,91 & 74,80 \\
$1.501-2.500 \mathrm{~g}$ & 4,77 & 9,87 & 24,01 & 42,87 & 62,16 \\
$>2.500 \mathrm{~g}$ & 4,59 & 11,46 & 25,00 & 42,69 & 61,33 \\
\hline
\end{tabular}


Tabela 17. Pacientes com infecções hospitalares e hemocultura positiva, segundo microrganismo isolado. Secretaria de Saúde, Estado de São Paulo, 2005.

\begin{tabular}{|c|c|c|}
\hline \multirow{2}{*}{ Microorganismo } & \multicolumn{2}{|c|}{ Pacientes com hemocultura positiva } \\
\hline & $\mathrm{N}$ & $\%$ \\
\hline Staphylococcus epidermidis e outros coagulase negativa & 2.540 & 29,91 \\
\hline Outros microrganismos & 1.622 & 19,10 \\
\hline S. aureus resistente à oxacilina & 856 & 10,08 \\
\hline S. aureus sensível à oxacilina & 833 & 9,81 \\
\hline Candida sp. & 477 & 5,62 \\
\hline Acinetobacter baumanii sensível ao imipenen & 366 & 4,31 \\
\hline Pseudomonas sp. sensível ao imipenem & 361 & 4,25 \\
\hline Escherichia coli sensível à cefalosporina de $3^{\text {a }}$ geração & 299 & 3,52 \\
\hline Klebsiella pneumoniae sensível à cefalosporina de $3^{\mathrm{a}}$ geração & 286 & 3,37 \\
\hline Pseudomonas sp. resistente ao imipenem & 252 & 2,97 \\
\hline K. pneumoniae resistente à cefalosporina de $3^{\mathrm{a}}$ geração & 213 & 2,51 \\
\hline Enterococcus sp. sensível à vancomicina & 197 & 2,32 \\
\hline A. baumanii resistente ao imipenen & 92 & 1,08 \\
\hline E. coli resistente à cefalosporina de $3^{\mathrm{a}}$ geração & 75 & 0,88 \\
\hline Enterococcus sp. resistente à vancomicina & 23 & 0,27 \\
\hline Total de pacientes com hemoculturas positivas & 8.492 & 100,00 \\
\hline
\end{tabular}

Tabela 18. Perfil de resistência dos microrganismos isolados em hemocultura de pacientes com infecções hospitalares. Secretaria da Saúde, Estado de São Paulo, 2005.

\begin{tabular}{|c|c|c|c|}
\hline Microorganismo & & Total & $\% *$ \\
\hline A. baumanii resistente ao imipenen & & 92 & 1,08 \\
\hline \multirow[t]{3}{*}{ A. baumanii sensível ao imipenen } & & 366 & 4,31 \\
\hline & Subtotal & 458 & \\
\hline & \% resistência & 20 & \\
\hline E. coli resistente à cefalosporina de $3^{\mathrm{a}}$ geração & & 75 & 0,88 \\
\hline \multirow[t]{3}{*}{ E. coli sensível à cefalosporina de $3^{\mathrm{a}}$ geração } & & 299 & 3,52 \\
\hline & Subtotal & 374 & \\
\hline & \% resistência & 20 & \\
\hline Enterococcus sp. sensível à vancomicina & & 197 & 2,32 \\
\hline \multirow[t]{3}{*}{ Enterococcus sp. resistente à vancomicina } & & 23 & 0,27 \\
\hline & Subtotal & 220 & \\
\hline & \% resistência & 10 & \\
\hline K. pneumoniae resistente à cefalosporina de $3^{\mathrm{a}}$ geração & & 213 & 2,51 \\
\hline \multirow[t]{3}{*}{ K. pneumoniae sensível à cefalosporina de $3^{\mathrm{a}}$ geração } & & 286 & 3,37 \\
\hline & Subtotal & 499 & \\
\hline & \% resistência & 43 & \\
\hline Pseudomonas sp. sensível ao imipenem & & 361 & 4,25 \\
\hline \multirow[t]{3}{*}{ Pseudomonas sp. resistente ao imipenem } & & 252 & 2,97 \\
\hline & Subtotal & 613 & \\
\hline & \% resistência & 41 & \\
\hline S. aureus sensível à oxacilina & & 833 & 9,81 \\
\hline \multirow[t]{3}{*}{ S. aureus resistente à oxacilina } & & 856 & 10,08 \\
\hline & Subtotal & 1.689 & \\
\hline & \% resistência & 51 & \\
\hline
\end{tabular}

*Percentual do total de microrganismos isolados $(\mathrm{N}=8.492)$ 